\title{
Early decongestive therapy versus high-flow nasal cannula for the prevention of adverse clinical events in patients with acute cardiogenic pulmonary edema
}

\author{
Sang Yong Om¹, Junho Hyun ${ }^{1}$, Kyung Hun $\mathrm{Nam}^{2}$, Sun Hack Lee ${ }^{1}$, Seung Min Song ${ }^{3}$, Jung Ae Hong ${ }^{1}$, \\ Sang Eun Lee ${ }^{1}$, Min-Seok Kim ${ }^{1}$ \\ ${ }^{1}$ Department of Cardiology, ${ }^{2}$ Department of Pulmonary and Critical Care Medicine, ${ }^{3}$ Department of Internal Medicine, Asan Medical Center, \\ University of Ulsan College of Medicine, Seoul, Korea \\ Contributions: (I) Conception and design: MS Kim; (II) Administrative support: KH Nam, SH Lee, SE Lee, MS Kim; (III) Provision of study \\ materials or patients: SE Lee, MS Kim; (IV) Collection and assembly of data: SY Om, JH Hyun; (V) Data analysis and interpretation: SY Om, MS \\ Kim; (VI) Manuscript writing: All authors; (VII) Final approval of manuscript: All authors. \\ Correspondence to: Min-Seok Kim, MD, PhD. Clinical Associate Professor, Department of Cardiology, Asan Medical Center, University of Ulsan \\ College of Medicine, 88, Olympic-ro 43-gil, Songpa-gu, Seoul 05505, Korea. Email: msk@amc.seoul.kr.
}

Background: Few studies have investigated the role of decongestive therapy and high-flow nasal cannula (HFNC) in preventing reintubation and in-hospital mortality in patients with acute cardiogenic pulmonary edema (ACPE).

Methods: Data from patients with ACPE who were weaned from mechanical ventilation in the cardiac intensive care unit between January 2013 and December 2017 were retrospectively evaluated. All patients were treated with HFNC or conventional oxygen therapy (COT), such as a nasal cannula or venturi mask, immediately after extubation. Decongestive therapy (intravenous furosemide infusion) was administered at the discretion of the attending physician.

Results: Of 212 patients treated during the study period, 47 were excluded due to recent open-heart surgery and two, due to insufficient clinical data. The remaining 163 patients had a mean age of $67.4 \pm 14.3$ years, and 92 (56.4\%) were male; 44 patients received HFNC, and 119 COT. Mean weight loss within 72 hours of extubation was $-0.86 \pm 2.03 \mathrm{~kg}$. A total of 38 patients $(23.3 \%)$ required reintubation, 21 of whom $(12.9 \%)$ required reintubation within 72 hours of extubation. In-hospital mortality occurred in 16 patients (9.8\%). Multivariate analysis showed that weight increase within 72 hours of extubation was independent determinants of reintubation ( $\mathrm{OR}=1.7 ; 95 \% \mathrm{CI}: 1.2-2.2 ; \mathrm{P}<0.001)$ and in-hospital mortality $(\mathrm{OR}=1.5 ; 95 \%$ CI: 1.1-2.1; $\mathrm{P}=0.005)$. The use of HFNC was not associated with reintubation or in-hospital mortality.

Conclusions: Our findings indicate that early weight loss resulted in reduced reintubation and in-hospital mortality in patients with ACPE. However, HFNC and COT did not differ in the prevention of reintubation and in-hospital mortality. Therefore, aggressive decongestive therapy, rather than HFNC, should be considered early after extubation.

Keywords: Acute cardiogenic pulmonary edema (ACPE); high-flow nasal cannula (HFNC); early decongestive therapy

Submitted Apr 15, 2019. Accepted for publication Aug 06, 2019.

doi: $10.21037 /$ jtd.2019.08.114

View this article at: http://dx.doi.org/10.21037/jtd.2019.08.114 


\section{Introduction}

In patients with acute heart failure (HF), acute cardiogenic pulmonary edema (ACPE) can develop as a result of increased pulmonary capillary hydrostatic pressure, resulting in fluid accumulation that cannot be cleared by lymphatic interstitial drainage (1). The rapid increase in interstitial and alveolar fluid leads to progressive respiratory failure. Over $90 \%$ of patients with acute HF present with fluid overload, primarily in the form of pulmonary congestion, which may lead to the requirement for mechanical ventilation (2).

Despite being successfully weaned from mechanical ventilation, a considerable number of patients require reintubation (3), and those with pre-existing heart disease or fluid accumulation are considered to be at higher risk (4). One study showed that up to $10 \%$ of patients with HF after planned extubation required reintubation (5). In general, patients who require reintubation have higher rates of inhospital mortality, increased intensive care unit (ICU) and hospital stays, prolonged duration of mechanical ventilation, higher medical costs, and a higher possibility of tracheostomy than patients who have a successful extubation $(3,6)$. However, an effective approach to preventing reintubation has not yet been determined in patients with ACPE.

Current evidence suggests that the use of non-invasive ventilation (NIV) can decrease the reintubation rate and ICU and hospital mortality rates in patients with planned extubation (7). It has also been seen to be effective in reducing the reintubation rate after cardiothoracic surgery $(8,9)$. In addition, positive intrathoracic pressure improves alveolar ventilation and leads to an increase in oxygenation and a further decrease in the work of breathing (10). Among various modalities providing positive intrathoracic pressure, high-flow nasal cannula (HFNC) is a simple method that provides continuous nasopharyngeal positive pressure with oxygen that exceeds patients' spontaneous inspiratory demand through a nasal cannula. However, data on the role of HFNC in patients with ACPE post-extubation are scarce.

In the present study, we aimed to determine whether HFNC might be superior to conventional oxygen therapy (COT) for preventing reintubation in successfully extubated patients with ACPE. In addition, predictors of better clinical outcomes after weaning from mechanical ventilation were investigated.

\section{Methods}

\section{Study population}

Observational data were collected retrospectively from the electronic medical records of patients with ACPE who were weaned from mechanical ventilation between January 2013 and December 2017 in the cardiac ICU of a largevolume single center in Korea. The study cohort included consecutive patients over 18 years old who received mechanical ventilatory support due to ACPE in the cardiac ICU and were extubated following clinical improvement. ACPE was diagnosed according to the algorithm of current guidelines, including signs and symptoms of HF, pulmonary edema on chest radiograph, and cardiac functional and structural abnormalities on echocardiography (2). Patients who were treated with mechanical ventilation due to noncardiogenic pulmonary edema, expired before extubation, or underwent recent open-heart surgery were excluded. All data were retrospectively collected by thorough chart review, using the hospital's electronic medical record system, including demographics, comorbidities, changes in body weight, Acute Physiology and Chronic Health Evaluation (APACHE) II score at ICU admission, mechanical ventilation time, and duration of ICU stay and hospital stay.

The study protocol was approved by the institutional review board. As no patient care intervention was required for this retrospective study, the requirement for informed consent was waived.

\section{Post-extubation oxygen therapy and fuid management during the hospital stay}

All patients were treated with HFNC or COT, such as nasal cannula or venturi mask, immediately after extubation, and intravenous furosemide infusion was initiated and maintained as decongestive therapy at the discretion of the attending physician considering the patients' volume status during the ICU and hospital stay.

\section{Study outcomes}

The primary outcomes of the present study were early reintubation and in-hospital mortality. Secondary outcomes included delayed reintubation, total reintubation, post- 


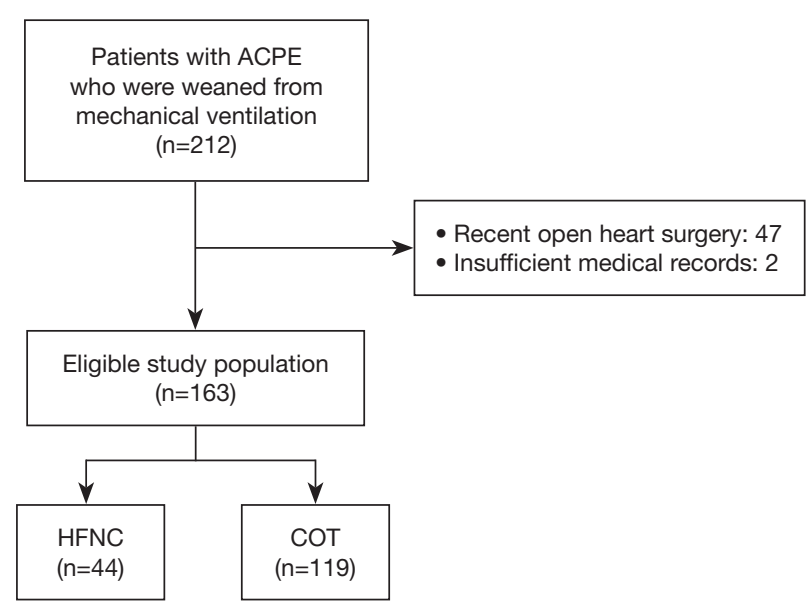

Figure 1 Flowchart of study population. Among the 212 patients, 163 patients were finally enrolled in this retrospective observational study. ACPE, acute cardiogenic pulmonary edema; HFNC, highflow nasal cannula; COT, conventional oxygen therapy.

extubation ICU and hospital length of stay, and respiratory rate at 1 hour after extubation. Early and delayed reintubation were defined as all-cause reintubation within 72 hours after extubation, and all-cause reintubation $>72$ hours from the time of extubation, respectively. All study outcomes were obtained using hospital electronic medical records.

\section{Statistical analysis}

The baseline characteristics of the study population, including patient demographics, risk factors or comorbidities, etiology of ICU admission, and arterial blood gas profiles before extubation, were compared between the HFNC group and the COT group. Continuous variables were expressed as mean \pm standard deviation or median [interquartile range (IQR)], depending on variable distribution. Categorical variables were presented as numbers and percentages. Group comparisons according to the methods of post-extubation oxygen therapy were analyzed using the Wilcoxon ranksum test for continuous variables, and the Chi-square test or Fisher's exact test for categorical variables. To minimize the potential differences in the baseline characteristics of patients treated with HFNC or COT, a propensity score (PS) matching method was utilized. Patients with ACPE were matched on PS at a 1:1 ratio according to the modalities for post-extubation oxygen therapy. PSs were estimated using a logistic model that included sex, age, body mass index, APACHE II score, duration of mechanical ventilation, $\mathrm{PaO}_{2} / \mathrm{FiO}_{2}$ ratio before extubation, $\mathrm{PaCO}_{2}$ before extubation, and left ventricular ejection fraction as covariates. Outcome parameters were also compared at baseline and after PS matching between the two groups. A logistic regression model was used to identify any factors that were independently associated with the primary outcomes.

All statistical analyses including PS matching method were performed using IBM SPSS Statistics 22.0 (IBM Corp., Armonk, NY, USA). A P value of $<0.05$ was considered statistically significant.

\section{Results}

\section{Baseline characteristics}

Between January 2013 and December 2017, 212 patients with ACPE were weaned from mechanical ventilation. Of these, 47 patients who underwent recent open-heart surgery, and two who had insufficient medical records, were excluded. A total of 163 consecutive patients with ACPE were finally enrolled (Figure 1).

Overall, the mean age of the study population was $67.4 \pm 14.3$ years and $92(56.4 \%)$ were male; 44 patients were treated with HFNC, and 119 patients with COT. The baseline demographic and clinical characteristics of the study population grouped according to treatment are summarized in Table 1. Patients in the HFNC group had a relatively longer duration of mechanical ventilation, lower $\mathrm{PaO}_{2} / \mathrm{FiO}_{2}$ ratio, and slightly higher $\mathrm{PaCO}_{2}$ before extubation than those in the COT group. There were no significant differences in echocardiographic parameters between the two groups. And the trend of patients' weight change during the ICU and hospital stay was shown in Figure S1.

\section{Primary and secondary outcomes}

During the study period, 38 patients $(23.3 \%)$ were reintubated. Among them, 21 patients (12.9\%) were reintubated within 72 hours of extubation; in-hospital death occurred in 16 patients $(9.8 \%)$. The primary outcomes did not differ significantly between the HFNC and COT groups (13.6\% vs. $12.6 \%$, respectively, in the early reintubation group, $\mathrm{P}=1.000$ and $13.6 \%$ vs. $8.4 \%$, respectively, in the in-hospital mortality group, $\mathrm{P}=0.375$; Table 2). Analyses of secondary outcomes showed no differences in the rates of delayed reintubation ( $\geq 72$ hours) and total reintubation, the duration of post-extubation ICU and hospital stay, and 
Table 1 Baseline patient demographic and clinical characteristics

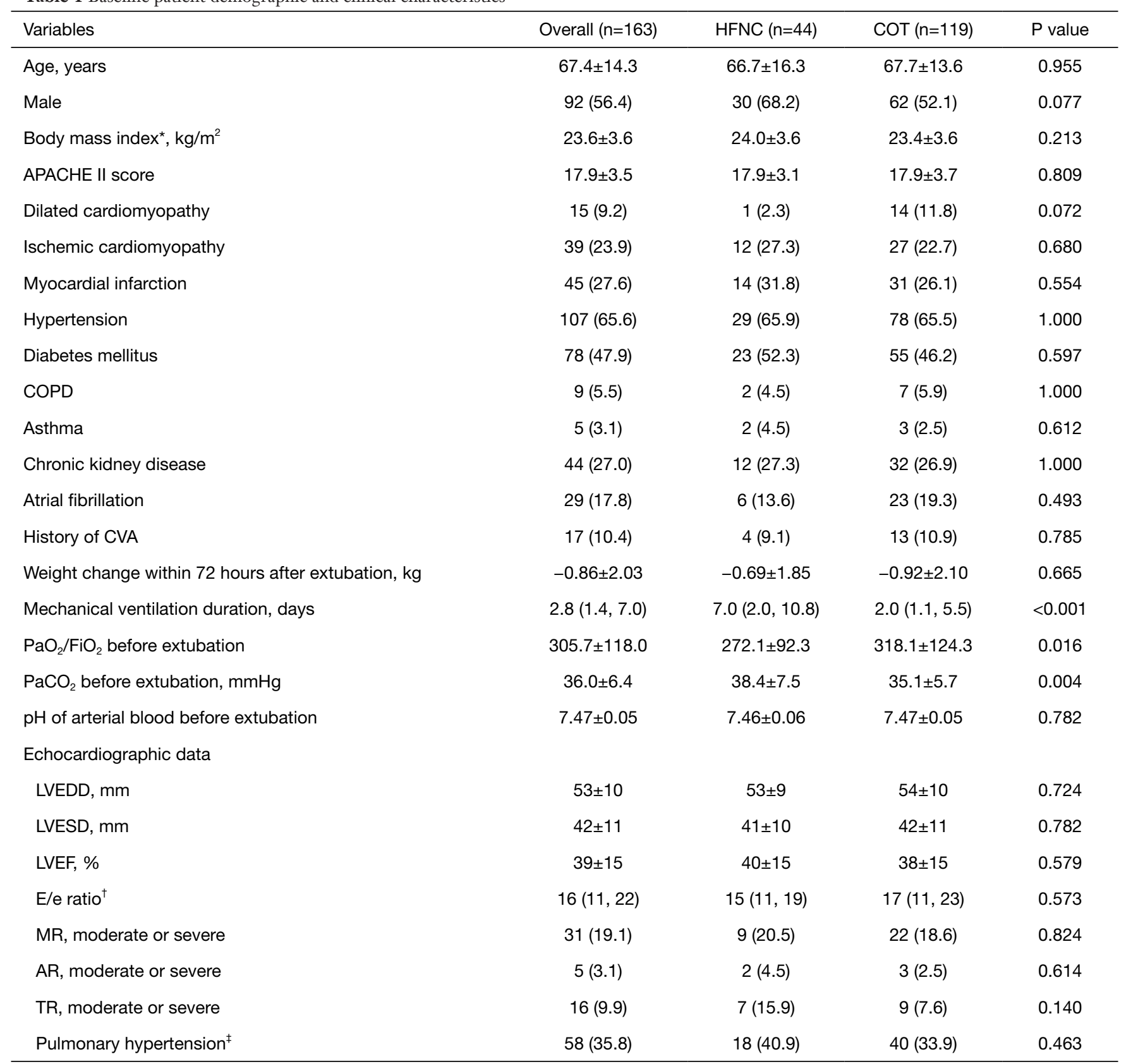

Data are expressed as number (\%), mean \pm standard deviation, or median (interquartile range), depending on variable distribution. *, body mass index is the weight in kilograms divided by the square of the height in meters; ${ }^{\dagger}$, E/e ratio is defined as the ratio of early diastolic mitral inflow (E) to early diastolic mitral annular tissue velocity (e); ${ }^{\ddagger}$, pulmonary hypertension indicates pulmonary artery systolic pressure $\geq 50 \mathrm{mmHg}$. APACHE, Acute Physiology and Chronic Health Evaluation; AR, aortic regurgitation; COPD, chronic obstructive pulmonary disease; CVA, cerebrovascular accident; EF, ejection fraction; LVEDD, left ventricular end-diastolic dimension; LVESD, left ventricular end-systolic dimension; MR, mitral regurgitation; TR, tricuspid regurgitation. 
Table 2 Primary and secondary outcomes

\begin{tabular}{|c|c|c|c|c|}
\hline Outcomes & Overall $(n=163)$ & HFNC $(n=44)$ & COT $(n=119)$ & $P$ value \\
\hline Early reintubation, $<72$ hours & $21(12.9)$ & $6(13.6)$ & $15(12.6)$ & 1.000 \\
\hline In-hospital mortality & $16(9.8)$ & $6(13.6)$ & $10(8.4)$ & 0.375 \\
\hline \multicolumn{5}{|l|}{ Secondary outcomes } \\
\hline Reintubation, total & $38(23.3)$ & $12(27.3)$ & $26(21.8)$ & 0.532 \\
\hline Post-extubation ICU stay*, days & $3.3(2.0,7.1)$ & $5.2(3.1,14.5)$ & $3.0(1.6,6.0)$ & 0.060 \\
\hline Post-extubation hospital stay*, days & $16.8(8.8,35.8)$ & $27.8(13.7,43.3)$ & $13.3(7.9,34.4)$ & 0.141 \\
\hline Respiratory rate at 1 hour after extubation, per minute & $21.9 \pm 5.9$ & $22.4 \pm 5.2$ & $21.7 \pm 6.2$ & 0.390 \\
\hline
\end{tabular}

${ }^{*}$, values are expressed as median (interquartile range).

respiratory rate at 1 hour after extubation according to the modalities for post-extubation oxygen therapy.

\section{PS matching analysis}

A total of 38 patients in the HFNC group were 1:1 PS matched to 38 patients in the COT group. The baseline characteristics of the PS matched cohorts are summarized in Table 3. There was no difference in all variables between the HFNC and the COT groups after PS matching. Standardized mean differences of all covariates are shown in Table S1. In the matched HFNC and COT cohorts, no significant differences were seen between the two groups in terms of the primary and secondary outcomes (Table 4).

\section{Factors influencing primary outcomes}

To determine any independent association between the post-extubation NIV modalities and primary outcomes, a multivariate logistic regression analysis was performed, adjusted for clinically relevant covariates (Table 5). After adjustment for a wide range of confounding factors, weight increase within 72 hours after extubation $(\mathrm{OR}=1.7$; $95 \% \mathrm{CI}$ : 1.2-2.2; $\mathrm{P}<0.001)$ and a history of hypertension $(\mathrm{OR}=0.3$; 95\% CI: $0.1-0.9 ; \mathrm{P}=0.035$ ) were seen to be independent determinants of early reintubation. Weight increase within 72 hours was also associated with in-hospital mortality ( $\mathrm{OR}=1.5 ; 95 \%$ CI: 1.1-2.1; $\mathrm{P}=0.005)$. However, the use of HFNC was not associated with total or early reintubation, or in-hospital mortality.

\section{Discussion}

In the present study, HFNC was not seen to be beneficial versus COT for preventing reintubation and in-hospital mortality in patients with ACPE. Similar results were seen after adjusting for clinical characteristics, including the degree of volume reduction. Early decongestive therapy within 3 days of extubation was a common determinant for reintubation or in-hospital mortality.

Our observations are not consistent with those of many previous studies of HFNC, which have generally demonstrated the benefit of this approach in reducing respiratory rate and improving oxygenation in critically ill patients with hypoxemic respiratory failure (11-13). However, most of the subjects in these studies had pneumonia or other respiratory diseases. One previous study of patients with ACPE demonstrated that HFNC decreased the respiratory rate compared with COT (14), but there is generally a paucity of data evaluating the use of HFNC in patients with ACPE, particularly in those weaned from mechanical ventilation.

There are several possible explanations for the lack of HFNC efficacy in post-extubation patients with ACPE seen in the current study. First, HF, which often induces $\mathrm{ACPE}$, is considered to be an independent risk factor for reintubation (15). One study showed HF to be the cause of $42 \%$ of failures of spontaneous breathing trials in medical ICU patients (16). Several studies have demonstrated that patients with ACPE have high rates of intubation and mortality, approximately $20 \%$ and $15 \%$, respectively (17-19). Post-extubation patients with ACPE appeared 
Table 3 Baseline demographic and clinical characteristics of propensity score matched patients

\begin{tabular}{|c|c|c|c|}
\hline Variables & HFNC $(n=38)$ & $\operatorname{COT}(n=38)$ & $P$ value \\
\hline Male & $25(65.8)$ & $23(60.5)$ & 0.812 \\
\hline Body mass index, $\mathrm{kg} / \mathrm{m}^{2}$ & $23.5 \pm 3.5$ & $23.7 \pm 3.5$ & 0.792 \\
\hline APACHE II score & $18.0 \pm 3.2$ & $17.8 \pm 3.9$ & 0.821 \\
\hline Ischemic cardiomyopathy & $11(28.9)$ & $8(21.1)$ & 0.597 \\
\hline Myocardial infarction & $11(28.9)$ & $8(21.1)$ & 0.597 \\
\hline Hypertension & $28(73.7)$ & $24(63.2)$ & 0.460 \\
\hline Diabetes mellitus & $20(52.6)$ & $14(36.8)$ & 0.249 \\
\hline Chronic kidney disease & $12(31.6)$ & $9(23.7)$ & 0.609 \\
\hline Atrial fibrillation & $5(13.2)$ & $12(31.6)$ & 0.097 \\
\hline History of CVA & $4(10.5)$ & $3(7.9)$ & 1.000 \\
\hline Weight change within 72 hours after extubation, kg & $-0.79 \pm 1.91$ & $-1.32 \pm 1.94$ & 0.239 \\
\hline Mechanical ventilation duration, days & $6.1 \pm 4.3$ & $6.3 \pm 4.0$ & 0.795 \\
\hline $\mathrm{PaO}_{2} / \mathrm{FiO}_{2}$ before extubation & $278.7 \pm 91.8$ & $262.7 \pm 85.3$ & 0.435 \\
\hline $\mathrm{PaCO}_{2}$ before extubation $(\mathrm{mmHg})$ & $37.3 \pm 7.3$ & $37.3 \pm 5.2$ & 0.971 \\
\hline $\mathrm{pH}$ of arterial blood before extubation & $7.46 \pm 0.06$ & $7.47 \pm 0.05$ & 0.919 \\
\hline MR, moderate or severe & $6(15.8)$ & $3(7.9)$ & 0.480 \\
\hline AR, moderate or severe & $2(5.3)$ & $0(0.0)$ & 0.493 \\
\hline $\mathrm{TR}$, moderate or severe & $6(15.8)$ & $2(5.3)$ & 0.262 \\
\hline Pulmonary hypertension ${ }^{\ddagger}$ & $15(39.5)$ & $8(21.1)$ & 0.133 \\
\hline
\end{tabular}

Data are expressed as number (\%), mean \pm standard deviation, or median (interquartile range), depending on variable distribution. ${ }^{\dagger}$, E/e ratio is defined as the ratio of early diastolic mitral inflow $(E)$ to early diastolic mitral annular tissue velocity (e). ${ }^{\ddagger}$, pulmonary hypertension indicates pulmonary artery systolic pressure $\geq 50 \mathrm{mmHg}$. APACHE, Acute Physiology and Chronic Health Evaluation; AR, aortic regurgitation; COPD, chronic obstructive pulmonary disease; CVA, cerebrovascular accident; EF, ejection fraction; LVEDD, left ventricular end-diastolic dimension; LVESD, left ventricular end-systolic dimension; MR, mitral regurgitation; TR, tricuspid regurgitation. 
Table 4 Primary and secondary outcomes of propensity score matched patients

\begin{tabular}{|c|c|c|c|}
\hline Outcomes & HFNC $(n=38)$ & COT $(n=38)$ & $P$ value \\
\hline Early reintubation, $<72$ hours & $5(13.2)$ & $4(10.5)$ & 1.000 \\
\hline In-hospital mortality & $6(15.8)$ & $2(5.3)$ & 0.262 \\
\hline \multicolumn{4}{|l|}{ Secondary outcomes } \\
\hline Reintubation, total & $11(28.9)$ & $6(15.8)$ & 0.271 \\
\hline Post-extubation ICU stay*, days & $5.0(3.0,13.5)$ & $3.0(1.8,7.0)$ & 0.054 \\
\hline Post-extubation hospital stay*, days & $27.0(13.0,43.3)$ & $13.5(9.5,37.0)$ & 0.087 \\
\hline Respiratory rate at 1 hour after extubation, per minute & $22.6 \pm 5.5$ & $22.7 \pm 6.1$ & 0.937 \\
\hline
\end{tabular}

${ }^{*}$, values are expressed as median (interquartile range).

Table 5 Multivariate regression analysis: independent factors for early reintubation (<72 hours) and in-hospital mortality

\begin{tabular}{lccc}
\hline \multirow{2}{*}{ Variables } & \multicolumn{2}{c}{ Early reintubation } & \multicolumn{2}{c}{ In-hospital mortality } \\
\cline { 2 - 3 } Weight change within 72 hours after extubation & OR $(95 \% \mathrm{Cl})$ & P value & OR $(95 \% \mathrm{Cl})$ \\
APACHE II score & $1.7(1.2-2.2)$ & $<0.001$ & $1.5(1.1-2.1)$ \\
Hypertension & $1.2(1.0-1.4)$ & 0.052 & 0.005 \\
History of asthma & $0.3(0.1-0.9)$ & 0.035 & 0.066 \\
Body mass index, $\mathrm{kg} / \mathrm{m}^{2}$ & $8.6(0.9-84.4)$ & & $0.8(0.7-1.0)$ \\
\hline
\end{tabular}

APACHE, Acute Physiology and Chronic Health Evaluation.

to be at higher risk of adverse clinical outcomes. In the current study, our patients showed relatively high rates of reintubation (23.3\%) and in-hospital mortality (9.8\%) compared with patients in previous studies and it is possible that the vulnerable nature of our study population may mitigate the benefit of HFNC.

Secondly, differences in modalities for post-extubation oxygen therapy may affect the results. Several meta-analyses have shown that NIV reduces the endotracheal intubation rate and tends to reduce mortality when compared with COT in patients with ACPE (17-19). Another large randomized trial revealed no differences in mortality between groups of patients receiving NIV versus COT (20). In general, HFNC appears to be better tolerated than other NIV modalities, including continuous positive airway pressure (CPAP) or non-invasive pressure support ventilation (NIPSV) (21). However, the level of positive pressure provided by HFNC is relatively low $\left(<5 \mathrm{cmH}_{2} \mathrm{O}\right)$ in comparison with CPAP or NIPSV. Moreover, the beneficial effect of continuous nasopharyngeal positive pressure by HFNC disappears when patients with ACPE breathe with an open mouth due to severe dyspnea. However, some compliance issues regarding the intolerance of HFNC was lacking in our study.

Finally, volume reduction is an important confounder as fluid overload is related to increased capillary leakage and extracellular volume distribution that can cause pulmonary edema. Consequently, this condition leads to impaired gas exchange, reduced compliance, and increased work of breathing (22). Some previous studies showed that a positive fluid balance was associated with failure to wean and a higher risk of extubation failure. The patients who failed to wean from mechanical ventilation due to a positive fluid balance had poor cardiovascular reserves $(22,23)$. These conditions can attenuate the effect of NIV in patients with ACPE, and strategies to manage fluid overload, such as the administration of diuretics or extracorporeal therapies, appear to play a key role in weaning success (24).

This study had certain limitations. First, the retrospective 
observational study design means that the setting and monitoring of oxygen therapy were dependent on the patients' attending physicians; differences in the HFNC setting could affect the failure rate of HFNC support, and the study results could possibly be affected by unknown confounders. Therefore, the interpretation of the results could be perverse, and the study is underpowered to show a difference in endpoints. The overall findings should be considered hypothesis-generating only. Secondly, some information regarding the application of HFNC was lacking, such as the oxygen dilution and entrainment of room air around the cannula. Also, the patients' compliance in the HFNC group is an important factor that may have influenced the outcomes. Thirdly, the sample size may have been inadequate and it is possible that analysis of a larger population is required to evaluate other ICU populations.

In conclusion, early decongestive therapy within 72 hours of extubation was independently associated with early reintubation and in-hospital mortality in patients with ACPE. However, HFNC and COT did not differ in preventing reintubation and in-hospital death. Therefore, aggressive decongestive therapy, rather than HFNC, should be considered early after weaning from mechanical ventilation. A large prospective randomized trial would be required to determine whether the modalities for postextubation oxygen therapy influence weaning success or failure.

\section{Acknowledgments}

None.

\section{Footnote}

Conflicts of Interest: The authors have no conflicts of interest to declare.

Ethical Statement: The authors are accountable for all aspects of the work in ensuring that questions related to the accuracy or integrity of any part of the work are appropriately investigated and resolved. The study was approved by the Local Bioethical Committee Asan Medical Center Institutional Review Board (2018-0161) and informed consent from the patients was waived.

\section{References}

1. Mebazaa A, Pang PS, Tavares M, et al. The impact of early standard therapy on dyspnoea in patients with acute heart failure: the URGENT-dyspnoea study. Eur Heart J 2010;31:832-41.

2. Ponikowski P, Voors AA, Anker SD, et al. 2016 ESC Guidelines for the diagnosis and treatment of acute and chronic heart failure: The Task Force for the diagnosis and treatment of acute and chronic heart failure of the European Society of Cardiology (ESC)Developed with the special contribution of the Heart Failure Association (HFA) of the ESC. Eur Heart J 2016;37:2129-200.

3. Thomrongpairoj P, Tongyoo S, Tragulmongkol W, et al. Factors predicting failure of noninvasive ventilation assist for preventing reintubation among medical critically ill patients. J Crit Care 2017;38:177-81.

4. Thille AW, Richard JC, Brochard L. The decision to extubate in the intensive care unit. Am J Respir Crit Care Med 2013;187:1294-302.

5. Hernandez G, Vaquero C, Colinas L, et al. Effect of Postextubation High-Flow Nasal Cannula vs Noninvasive Ventilation on Reintubation and Postextubation Respiratory Failure in High-Risk Patients: A Randomized Clinical Trial. JAMA 2016;316:1565-74.

6. Thille AW, Harrois A, Schortgen F, et al. Outcomes of extubation failure in medical intensive care unit patients. Crit Care Med 2011;39:2612-8.

7. Lin C, Yu H, Fan H, et al. The efficacy of noninvasive ventilation in managing postextubation respiratory failure: a meta-analysis. Heart Lung 2014;43:99-104.

8. Olper L, Corbetta D, Cabrini L, et al. Effects of noninvasive ventilation on reintubation rate: a systematic review and meta-analysis of randomised studies of patients undergoing cardiothoracic surgery. Crit Care Resusc 2013;15:220-7.

9. Zhu G, Huang Y, Wei D, et al. Efficacy and safety of noninvasive ventilation in patients after cardiothoracic surgery: A PRISMA-compliant systematic review and meta-analysis. Medicine (Baltimore) 2016;95:e4734.

10. Pham T, Brochard LJ, Slutsky AS. Mechanical Ventilation: State of the Art. Mayo Clin Proc 2017;92:1382-400.

11. Sztrymf B, Messika J, Bertrand F, et al. Beneficial effects of humidified high flow nasal oxygen in critical care patients: a prospective pilot study. Intensive Care Med 2011;37:1780-6.

12. Sztrymf B, Messika J, Mayot T, et al. Impact of highflow nasal cannula oxygen therapy on intensive care unit patients with acute respiratory failure: a prospective observational study. J Crit Care 2012;27:324.e9-13.

13. Schwabbauer N, Berg B, Blumenstock G, et al. Nasal high- 
flow oxygen therapy in patients with hypoxic respiratory failure: effect on functional and subjective respiratory parameters compared to conventional oxygen therapy and non-invasive ventilation (NIV). BMC Anesthesiol 2014;14:66.

14. Makdee O, Monsomboon A, Surabenjawong U, et al. High-Flow Nasal Cannula Versus Conventional Oxygen Therapy in Emergency Department Patients With Cardiogenic Pulmonary Edema: A Randomized Controlled Trial. Ann Emerg Med 2017;70:465-72.e2.

15. Maggiore SM, Battilana M, Serano L, et al. Ventilatory support after extubation in critically ill patients. Lancet Respir Med 2018;6:948-62.

16. Cabello B, Thille AW, Roche-Campo F, et al. Physiological comparison of three spontaneous breathing trials in difficult-to-wean patients. Intensive Care Med 2010;36:1171-9.

17. Masip J, Roque M, Sanchez B, et al. Noninvasive ventilation in acute cardiogenic pulmonary edema: systematic review and meta-analysis. JAMA 2005;294:3124-30.

18. Peter JV, Moran JL, Phillips-Hughes J, et al. Effect of non-invasive positive pressure ventilation (NIPPV) on mortality in patients with acute cardiogenic pulmonary

Cite this article as: Om SY, Hyun J, Nam KH, Lee SH, Song SM, Hong JA, Lee SE, Kim MS. Early decongestive therapy versus high-flow nasal cannula for the prevention of adverse clinical events in patients with acute cardiogenic pulmonary edema. J Thorac Dis 2019;11(9):3991-3999. doi: 10.21037/ jtd.2019.08.114 oedema: a meta-analysis. Lancet 2006;367:1155-63.

19. Winck JC, Azevedo LF, Costa-Pereira A, et al. Efficacy and safety of non-invasive ventilation in the treatment of acute cardiogenic pulmonary edema--a systematic review and meta-analysis. Crit Care 2006;10:R69.

20. Gray A, Goodacre S, Newby DE, et al. Noninvasive ventilation in acute cardiogenic pulmonary edema. N Engl J Med 2008;359:142-51.

21. Frat JP, Brugiere B, Ragot S, et al. Sequential application of oxygen therapy via high-flow nasal cannula and noninvasive ventilation in acute respiratory failure: an observational pilot study. Respir Care 2015;60:170-8.

22. Claure-Del Granado R, Mehta RL. Fluid overload in the ICU: evaluation and management. BMC Nephrol 2016;17:109.

23. Liu Y, An Z, Chen J, et al. Risk factors for noninvasive ventilation failure in patients with post-extubation acute respiratory failure after cardiac surgery. J Thorac Dis 2018;10:3319-28.

24. Ghosh S, Chawla A, Mishra K, et al. Cumulative Fluid Balance and Outcome of Extubation: A Prospective Observational Study from a General Intensive Care Unit. Indian J Crit Care Med 2018;22:767-72. 


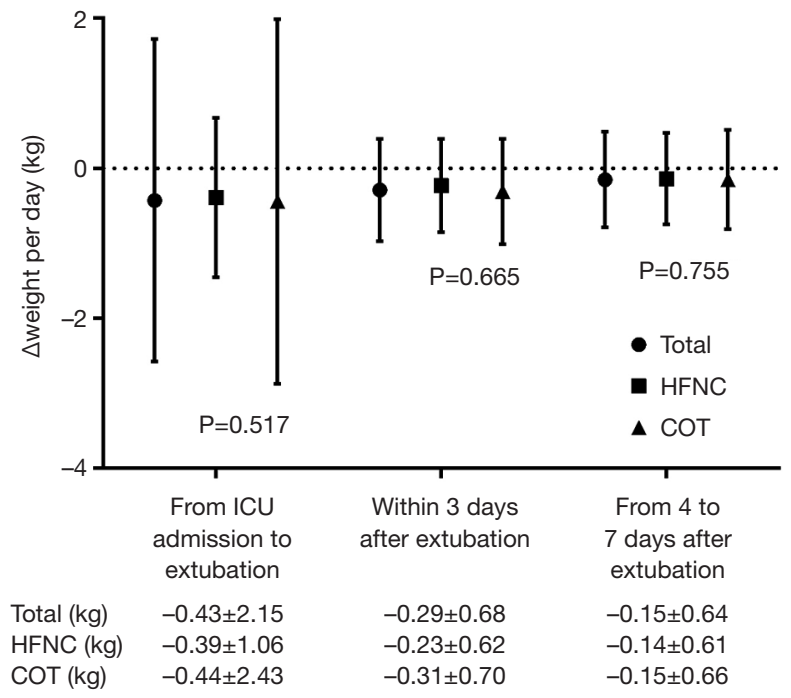

Figure S1 The trend of patients' weight change during the ICU and hospital stay. ICU, intensive care unit; HFNC, high-flow nasal cannula; COT, conventional oxygen therapy.

Table S1 Standardized mean difference of co-variables before and after propensity score matching

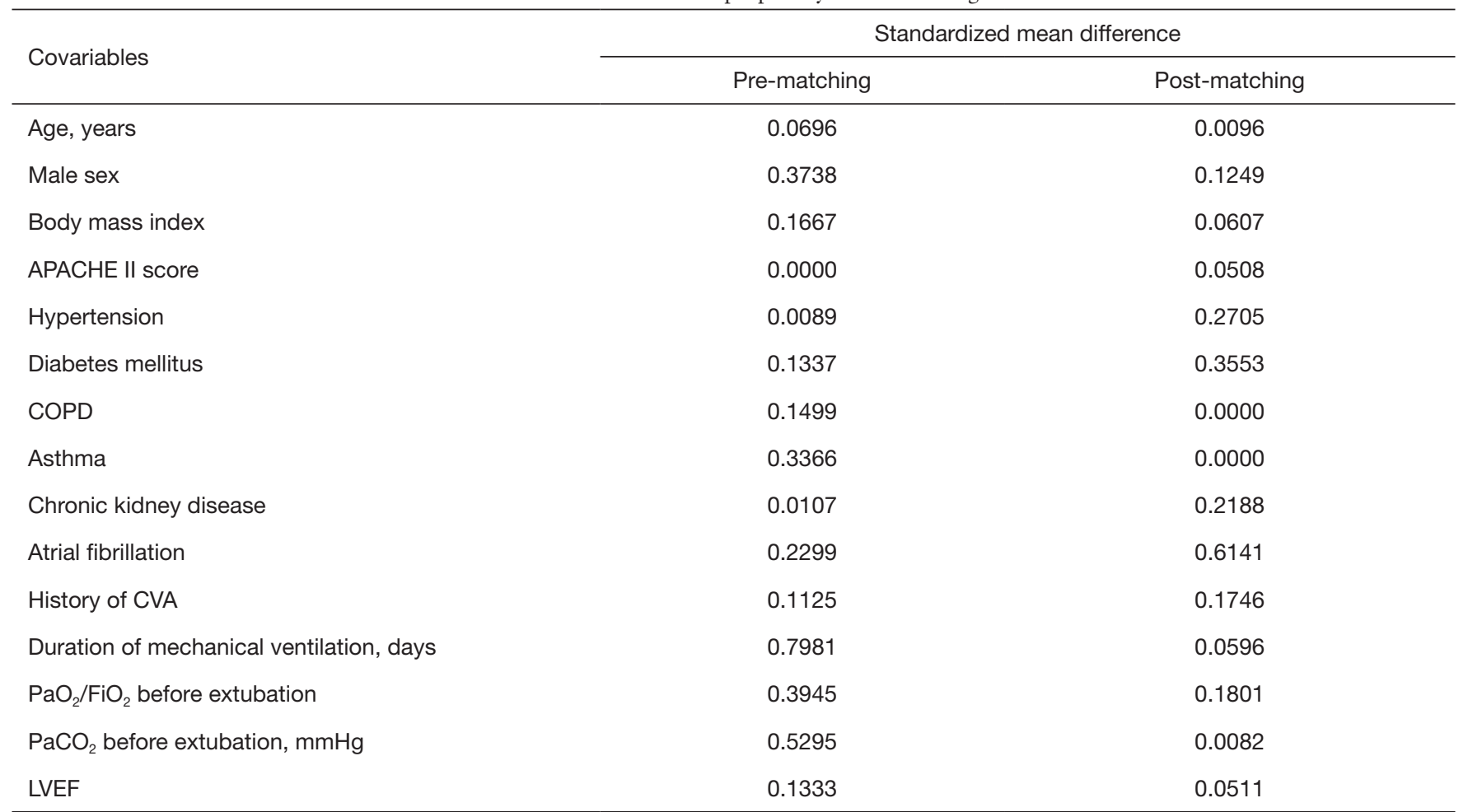

APACHE, Acute Physiology and Chronic Health Evaluation; COPD, chronic obstructive pulmonary disease; CVA, cerebrovascular accident; LVEF, left ventricular ejection fraction. 